Ann. Génét. Sél. anim., 1969, 1 (3), 299-301.

\title{
HÉRITABILITÉS ET INTERACTIONS GÉNOTYPE X RÉGIME POUR LE POIDS A HUIT SEMAINES DE POULETS EN PRÉSENCE DE DEUX ALIMENTS
}

\author{
P. MÉRAT et Jacqueline PROD'HOMME \\ Station centrale de Génétique animale, \\ Station de Recherches avicoles, \\ Centre national de Recherches zootechniques, 78-Jouy-en-Josas \\ Institut national de la Recherche agronomique
}

En I 962,1 'héritabilité du poids à 8 semaines et de plusieurs critères de conformation a été comparée pour deux groupes d'animaux d'une même souche ( $J 66$, sélectionnée pour la vitesse de croissance) recevant ad libitum un aliment de teneur différente en énergie et protéines. Chez les femelles, l'héritabilité de ces caractères était plus élevée en présence de 1'aliment le plus riche (BOYER, DE LAAGE et CALET, I963).

En mai-juin de la même année, une comparaison semblable était faite sur une autre population " synthétique " ( $J$ 44) sélectionnée pour plusieurs caractères, dont la croissance. Sur 8 éclosions successives, celles de rang impair recevaient une ration plus riche en protéines et énergie (aliment " enrichi ") que celles de rang pair (aliment " basse énergie "). Un anticoccidien était adjoint au premier aliment.

Les effectifs étaient voisins de 800 poussins par sexe et par régime, avec 20 pères et environ 8 mères par père.

Le tableau $\mathrm{I}$ indique les valeurs moyennes du poids à 8 semaines pour chaque groupe.

TABLEAU I

Poids moyens à 8 semaines par sexe et régime

\begin{tabular}{|c|c|c|}
\hline \multirow{2}{*}{ sexe } & \multicolumn{2}{|c|}{ poids (g) } \\
\hline & $\begin{array}{l}\text { Régime "basse } \\
\text { énergie " }\end{array}$ & Régime " enrichi " \\
\hline 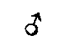 & 803 & 989 \\
\hline$q$ & 675 & 810 \\
\hline
\end{tabular}


La variance du poids semble légèrement plus élevée dans chaque sexe en présence de l'aliment " enrichi ". Les distributions de fréquences sont un peu dissymétriques, avec l'un ou l'autre régime.

Les différences les plus intéressantes concernent 1'héritabilité, augmentée dans les deux sexes avec l'aliment "enrichi " (tabl. 2). Nous désignons par $h_{\mathbf{D}}^{2}$ l'héritabilité estimée à partir de la composante "mère " de la variance dans une classification hiérarchique par familles de frères-sœurs et demi-frères, demi-sœurs; $h_{\mathrm{s}}^{2}$ représente celle basée sur la composante " père ", et $h^{2}$ la moyenne des deux.

TABLEAU 2

Héritabilité du poids à 8 semaines suivant le type d'aliment

\begin{tabular}{|c|c|c|c|c|c|c|}
\hline \multirow{2}{*}{ Régime } & \multicolumn{3}{|c|}{$\partial \sigma^{0}$} & \multicolumn{3}{|c|}{ \$O } \\
\hline & $h_{\mathrm{D}}^{2}$ & $h_{\mathrm{S}}^{2}$ & $h^{2}$ & $h_{\mathrm{D}}^{2}$ & $h_{\mathrm{S}}^{2}$ & $h^{2}$ \\
\hline Enrichi $\ldots \ldots \ldots$ & I, 37 & 0,19 & 0,78 & $0,9 \mathrm{I}$ & 0,30 & 0,60 \\
\hline Basse énergie & 0,73 & 0,04 & $0,3^{8}$ & $0,7 \mathrm{I}$ & 0,25 & 0,49 \\
\hline
\end{tabular}

Nous avons, d'autre part, testé les interactions entre les facteurs " famille", " sexe " et " régime ", par une analyse de variance sur une fraction échantillonnée des données, avec trois enfants de chaque sexe choisis au hasard pour chaque mère et chaque régime (tabl. 3).

\section{TABLEAU 3}

Analyse de variance du poids à 8 semaines

(données échantillonnées)

\begin{tabular}{|c|c|c|c|c|}
\hline Source de variation & $\begin{array}{c}\text { Degrés de } \\
\text { liberté }\end{array}$ & Variance & $\mathbf{F}$ & Signification* \\
\hline 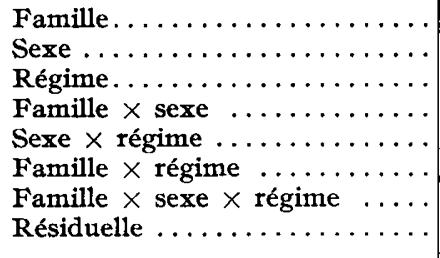 & $\begin{array}{r}50 \\
I \\
I \\
50 \\
I \\
50 \\
50 \\
404\end{array}$ & $\begin{array}{rr}29 & 475 \\
3640 & 250 \\
3939 & 255 \\
9 & 397 \\
99 & 400 \\
7 & 675 \\
7 & 975 \\
9 & 450\end{array}$ & $\begin{array}{r}3, \text { I I } \\
385,01 \\
416,63 \\
0,99 \\
10,51 \\
0,81 \\
0,84 \\
\end{array}$ & $\begin{array}{l}\mathrm{P}<0, \text { oOI } \\
\mathrm{P}<0,001 \\
\mathrm{P}<\mathrm{o,O01} \\
\mathrm{N} . \mathrm{S} . \\
\mathrm{P}<\mathrm{O,OI} \\
\text { N.S. } \\
\quad \text { N.S. } \\
\quad-\end{array}$ \\
\hline
\end{tabular}

* Nous avons testé les effets principaux, quoique leur interprétation soit compliquée par le fait que l'une des variances interaction est significative. 
On voit que la seule interaction significative est celle entre sexe et régime, les mâles bénéficiant davantage de l'aliment enrichi. En particulier, il n'y a pas d'interaction décelable entre famille et régime.

Comme il y avait ségrégation dans certaines familles aux loci $\mathrm{C}$ (blanc récessif) et $\mathrm{B} l$ (dilution du noir), nous avons testé, dans des sous-échantillons, et séparément pour chaque sexe, les effets principaux et interactions des facteurs " famille ", "génotype " (au locus envisagé) et " régime ". Dans les deux cas, le génotype ne présente ni effet principal significatif, ni interaction décelable, à l'exception d'une interaction famille $\times$ génotype $\times$ régime, significative au seuil I p. roo pour le gène $B l$ chez les femelles, d'interprétation difficile.

Le résultat sur les héritabilités du poids à 8 semaines paraît confirmer celui de BOYER et al. (I963). En outre, des données analogues ont été recueillies en I963 sur la souche J 66 avec deux aliments différant davantage par leur taux énergétique (BOYER, données non publiées) et chez deux sélectionneurs sur deux souches lourdes sélectionnées pour la croissance : " $V I I$ ” et " $T 22$ " (PROD' HOMmE, données non publiées).

Dans ces trois troupeaux, de même, l'héritabilité correspondant tant à la composante " mère " qu'à la composante " père " est augmentée en présence de l'aliment enrichi (dans les deux sexes, sauf pour la souche $T 22$ où seules les données sur les femelles étaient disponibles).

Ce type d'aliment paraît donc susceptible de permettre un gain génétique plus important par génération de sélection. L'absence d'interaction entre familles et régime suggère que - $\mathrm{du}$ moins dans la souche $J 44-$ cette sélection donne un résultat aussi en présence d'un aliment moins riche. Dans la souche $J 66$, la variance interaction est significative, au seuil 5 p. Ioo en I963, mais elle ne représente qu'une fraction assez réduite de la variance totale (PROD'Homme, données non publiées).

Reçu pour publication en novembre 1968 .

\author{
SUMMARY \\ HERITABILITIES AND GENOTYPE X DIET INTERACTIONS FOR 8-WEERS WEIGHT \\ OF CHICKEN WITH TWO DIFFERENT FEED TYPES
}

For the strain " $J 44 *$ in 1962, the heritability of 8-week weight, estimated from sire and dam components of variance in a hierarchical classification by full and half-sib families, was found higher in both sexes for a sample fed a high-protein, high energy feed than for a group receiving a feed with lower protein and energy. Published (Boyer et al. 1963) and unpublished data show analogous results in 3 other strains.

There was no significant interaction between family and feed type.

\title{
RÉFÉRENCE BIBLIOGRAPHIQUE
}

BOyer J.-P., DE LAAGE X., CALET C., r963. Influence of feed on the heritability of some chicken traits. $X I$. Int. Conf. Genet., 1, 262 (Abstr.). 